\title{
Deep sea megafaunal assemblages off the northern Iberian slope related to environmental factors
}

\author{
ALBERTO SERRANO, FRANCISCO SÁNCHEZ, ANTONIO PUNZÓN, \\ FRANCISCO VELASCO and IGNACIO OLASO
}

Instituto Español de Oceanografía de Santander, P.O. Box 240, 39080, Santander, Spain. E-mail: aserrano@st.ieo.es

\begin{abstract}
SUMMARY: Four deep sea fish and invertebrate assemblages from the northern Spanish slope were identified and related to geographic and bathymetric factors. The Peñas Cape comprises a boundary area splitting the more productive, wider and sandy western shelf from the less productive, narrow and muddy eastern part. The four faunal assemblages described were a shelf-slope transition assemblage (SST, 400-500 m); two upper slope assemblages $(500-650 \mathrm{~m}$ ), one corresponding to the western area of Cape Peñas and one to the eastern area; and a middle slope assemblage (650-750 $\mathrm{m})$. The SST was dominated by plankton-feeding fish and was hence less diverse than the other strata. This dominance was more evident west of the Peñas Cape, where upwelling was stronger. Regarding invertebrates, plankton-feeding fish and benthos-feeding invertebrates dominated the SST, whereas zooplankton-feeding and deposit-feeding invertebrates dominated the middle slope. The western assemblages were richer in species as a consequence of a more complex food web, especially of guilds related to water column production: plankton-feeding fish and filter- and zooplankton-feeding invertebrates.
\end{abstract}

Keywords: deep-sea, slope, spatial distribution, megafaunal communities, demersal species, epibenthos, trophic guilds.

RESUMEN: AGRUPACIONES MEGAFAUNALES DEL MAR PROFUNDO DEL TALUD NORTE ESPAÑOL EN RELACIÓN A FACTORES AMBIENTALES. - Se han identificado cuatro agrupaciones de peces e invertebrados en el talud norte español, relacionados con factores geográficos y batimétricos. Cabo Peñas representa una frontera que separa la plataforma occidental, más productiva, ancha y arenosa, de la oriental, menos productiva, más estrecha y de fondos fangosos. Las agrupaciones faunísticas descritas son: una agrupación de transición plataforma-talud (SST, 400-500 m), dos agrupaciones del talud superior (500-650 m), una correspondiendo con la zona occidental de Cabo Peñas (USW) y la otra con la zona oriental respecto al cabo (USE), y una agrupación del talud medio (MS, 650-750 m). La agrupación SST está dominada por peces planctófagos y presenta por ello menor diversidad que otros estratos. Esta dominancia es mayor al oeste de Cabo Peñas, donde los procesos de afloramiento son más fuertes. Respecto a los invertebrados, las especies bentófagas dominan en la agrupaciones SST, mientras que los depositívoros dominan en el talud medio. Las agrupaciones situadas al oeste presentan una mayor riqueza específica, como consecuencia de una red trófica más compleja, especialmente de grupos tróficos relacionados con la producción de la columna de agua: peces planctófagos e invertebrados filtradores o zooplanctófagos.

Palabras clave: mar profundo, talud, distribución espacial, comunidades megafaunales, epibentos, categorías tróficas.

\section{INTRODUCTION}

The deep-sea ecosystem has been traditionally described as an environment with a high environmental stability (Sanders, 1968; Stuart et al., 2003). This description has been modified by recent studies that have shown that species diversity and composition vary locally, regionally and even globally, suggesting changes at all spatial scales in environmental factors determining community structure (Grassle and Maciolek, 1992; Rex et al., 1993; Stuart et al., 2003). Food input represents the main way in which environmental changes affect deep sea communities (Gage, 2003). It is widely described that deep sea environments are heterotrophic, oligotrophic and allochthonous systems (Dayton and Hessler, 1972; Gage, 2003; Carney, 2005), since the low rate of food supply comes from the flux of organic-material to deep ocean basins from 


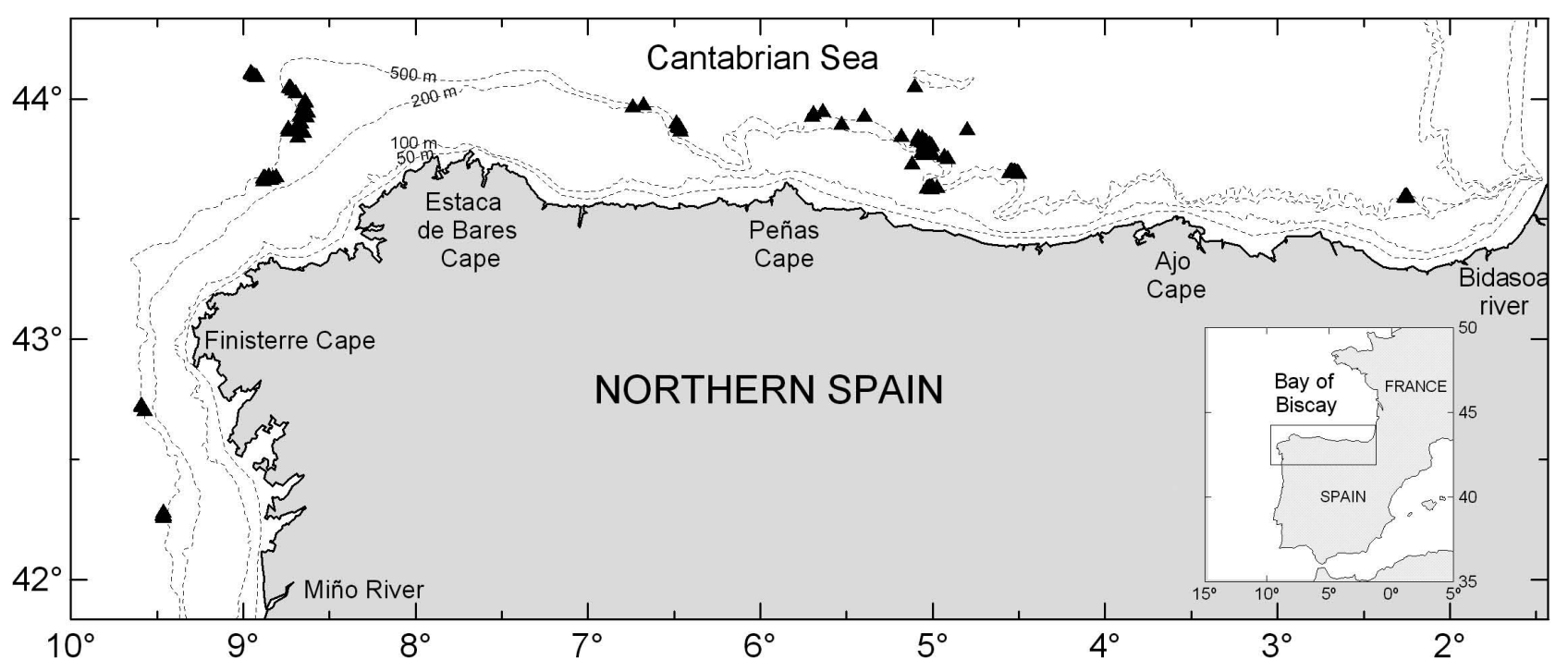

FIG. 1. - Study area. Black triangles represent haul locations.

the euphotic zone (surface primary production). The deep sea is subject to considerable temporal and spatial fluctuations in the pulses of organic matter derived from vertical and/or advective fluxes (e.g. nepheloid layers, submarine canyons).

The northern Spanish shelf is a good area to test how differences in environmental drivers may be reflected in the composition of deep sea fauna, since strong geographical differences in nutrient flux dynamics are found (see Study Area section). For instance, the strength of seasonal upwelling (Gil, 2008), estuarine outwelling (López-Jamar et al., 1992) and canyon funnelling (Sorbe, 1999) is particularly different between western (Atlantic) and eastern areas (Cantabrian Sea). Moreover, several studies have highlighted regional differences regarding other factors such as width and steepness of the shelf, and type of sediment (Rey and Medialdea, 1989; Serrano et al., 2006, 2008).

Deep waters of the northern Spanish continental shelf are under considerable fishing pressure, since Spanish fishermen have started to trawl deeper, increasing the effort of longline gears targeting deep species such as Phycis blennoides (Piñeiro et al., 2001). These fishing activities affect vulnerable species such as deep-water sharks and Bericiformes fish and also habitats inhabited by vulnerable invertebrates such as cnidarians and sponges (Sánchez et al., 2008). The management of deep-water fisheries off the northern coast of Spain requires a fundamental knowledge of the habitats that are being exploited by Spanish fishermen. However, studies on deep-sea ecosystem functioning in the area are scarce and geographically fragmented, and mainly focus on specific zones (Cartes et al., 2007; Sánchez et al., 2008; Preciado et al., 2009).

The aim of this study was to characterize the deep sea faunal assemblages of the northern Iberian slope and to determine how certain environmental factors affect their spatial distribution patterns. To answer these questions we tried to ascertain whether geographical differences in abiotic factors were reflected in different deep sea fauna distribution and composition.

\section{MATERIALS AND METHODS}

\section{Study area}

The study area includes the northern Spanish slope (400-750 m depth), between the verticals of the Rivers Miño and Bidasoa (Fig. 1). Geographically, the area is divided into two subareas: the Galician Atlantic slope from the River Miño to Cape Estaca de Bares, and the Cantabrian Sea slope between this cape and the River Bidasoa, at the beginning of the French shelf.

In addition to this geographical division marked by Cape Estaca de Bares, Cape Peñas has been described as an important environmental boundary in the area, from both the geological and the oceanographic point of view. The production in the area is greatly influenced by a seasonal coastal upwelling (spring and summer) and hydrographical mesoscale activity along the northwestern shelf-break (Gil, 2008). Lavín et al. (2005) report that upwelling events are more common and intense to the west of Cape Peñas, and that this is a mechanism of spatial variability between the western and eastern parts of the Cantabrian Sea. This is a consequence of the geographical location of the western area, which is closer than the eastern one to the anticyclone edge of the Azores High, and hence more influenced by eastern winds (Gil, 2008). Because of this, coastal summer upwelling strength - and hence chlorophyll values - decrease eastwards (Gil, 2008), and following the trophic webs these differences are also reflected in the abundance of zooplankton (Lavín et al, 2005). In spring (as a consequence of mountain snow melting), the southeasternmost corner of the Bay of Biscay is the area of greatest discharge of continental fresh water, mainly through French rivers. 
During this season, a boundary between the warm salty western water and the cold fresh eastern water is located around Cape Peñas (Gil, 2008).

There were also topographical differences between these two areas. One key factor is the different geomorphology of the two shelves (Rey and Medialdea, 1989). East of Cape Peñas, the Cantabrian sea shelf is very narrow (10-35 km), with an abrupt shelf break where the environmental gradients are very short, whereas west of Cape Peñas the shelf is wider $(25-75 \mathrm{~km})$, with a gradual transition between shelf and slope.

On the Galician shelf, the outwelling of large estuaries called rias is a very important process (LópezJamar et al., 1992), whereas rivers are not important on the Cantabrian shelf, where the role of great submarine canyons is more determinant (Sorbe, 1999). The sediment distribution in the two areas also differs: the Galician upper slope is composed mainly of sand (Serrano et al., 2008), while the Cantabrian upper slope consists mainly of mud (Serrano et al., 2006). The organic inputs of the French rivers thus lead to the presence of organic-enriched mud in the easternmost part of the Cantabrian Sea.

\section{Data source}

Bottom trawl surveys have been conducted every autumn (September-October) since 1983 by the Instituto Español de Oceanografía (IEO) to evaluate the status of demersal and megaepibenthic ecosystems on the northern Spanish shelf. The sampling unit consisted of 30-minute hauls at a speed of 3.0 knots, using a Baca 44/60 otter trawl gear (Sánchez and Serrano, 2003). The mesh size was $60 \mathrm{~mm}$ for the net and $10 \mathrm{~mm}$ for the cod end, which caught both demersal and megaepibenthic fauna. The mean horizontal opening was $18.9 \mathrm{~m}$ and the vertical opening was $2.0 \mathrm{~m}$. The otter trawl was monitored using a Scanmar net control system.

The time series used in the present study started in 1992, when all species - not only commercial oneswere systematically identified and quantified. Recording of oceanographic data using a Seabird CTD (bottom temperature and salinity) also started in 1992. In the present study, only hauls deeper than 400 m were considered as deep sea samples. The number of deepwater hauls increased yearly until 1992, when it was fixed at around 15 hauls per year. Figure 1 shows that the slopes between Cape Finisterre and Cape Estaca, and between Cape Peñas and Cape Ajo were the regions with the highest number of hauls, whereas the regions between the River Miño and Cape Finisterre, between Cape Estaca and Cape Peñas, and between Cape Ajo cape and the River Bidasoa were sampled less intensely, mainly due to the rougher seabed.

\section{Spatial distribution patterns of faunal assemblages}

For all multivariate analyses, the demersal and benthic fish and invertebrate species density matrix was reduced, considering only those species with a density greater than $0.04 \%$ and an occurrence greater than $5 \%$, in order to reduce the variability in the matrix due to the high presence of zeros. This reduced matrix was log-transformed.

To detect spatial patterns, a cluster analysis was applied to the species abundance matrix using the Bray-Curtis similarity index. The distance matrix was processed using the UPGMA algorithm. The nomenclature adopted for the assemblage groups was based on classifications adopted in several deep sea studies (Gordon, 1986; Haedrich and Merrett, 1988; Cartes et al., 1994), with slight modifications due to regional characteristic on shelf-slope topography (Rey and Medialdea, 1989; Sánchez and Serrano, 2003).

The effects of environmental variables on the assemblages were assessed using Redundancy Analysis (RDA) (Jongman et al., 1987). The abiotic variables used in the analysis were depth, near-bottom temperature, near-bottom salinity, geographical location (longitude) and sediment characteristics, including mean particle diameter $\left(\mathrm{Q}_{50}\right)$, sorting coefficient $\left(\mathrm{S}_{0}\right)$, weight percentage of gravel and coarse sands $(>500$ $\mu \mathrm{m})$, of medium, fine and very fine sands (63-500 $\mu \mathrm{m})$, and silt $(<63 \mu \mathrm{m})$, and weight percentage of organic matter. Year (survey) was included in the analysis as a covariable, to avoid inter-year (climatic) variability. Sediment was collected using a sediment collector attached to the groundrope. Particle size analysis of sediments was performed by a combination of dry sieving and sedimentation techniques (Buchanan, 1984). Organic matter in the sediment was estimated as weight loss of dried $\left(100^{\circ} \mathrm{C}, 24 \mathrm{~h}\right)$ samples after combustion $\left(500^{\circ} \mathrm{C}, 24 \mathrm{~h}\right)$. Temperature and salinity in the water column were also measured during each haul using a CTD Seabird SBE-911 (only near-bottom $\mathrm{T}$ and $\mathrm{S}$ were used).

The representativeness of the ordination analysis is given in terms of eigenvalues of the axes and variance explained by the biplots. The statistical significance was calculated with the Monte Carlo test (Verdonschot and Ter Braak, 1994) using 999 permutations under the reduced model. The significance of each environmental factor was tested by running the analysis with it as the variable and the other factors as covariables, and again calculated with the Monte Carlo test. Significance of groups obtained in the cluster analysis was also tested using the RDA model. Details of the model and procedure are described in Table 4.

RDA results are presented graphically in a bidimensional ordination diagram generated by biplot scaling focussed on inter-species distances, in which species are represented by points and environmental variables by vectors. The arrows of the explanatory variables indicate the direction of maximum change of these variables across the diagram. The projection of species categories onto an arrow gives an approximation of their weighted averages with respect to the variable. 
TABLE 1. - List of fish species identified in the area. with their density (ind. ha ${ }^{-1}$ ) in each assemblage. Species codes used in Figure $3 \mathrm{~b}$ appear between brackets after species name. in those species used in RDA analysis. 0.0 mean values $>0$ and $<0.1$

\begin{tabular}{|c|c|c|c|c|c|c|c|c|c|}
\hline & SST & USW & USE & MS & & SST & USW & USE & MS \\
\hline Chondrichthyes & & & & & Gonostoma bathyphilum & & & & 0.1 \\
\hline Centroscymnus coelolepis & & & 0.0 & 0.1 & Halargyreus johnsonii (Hjoh) & 0.1 & 0.8 & 3.7 & 3.2 \\
\hline Chimaera monstrosa (Cmon) & 14.2 & 16.2 & 2.0 & 53.5 & Halosaurus ovenii & & & & 0.2 \\
\hline Dalatias licha & 0.0 & 0.1 & 0.0 & & Helicolenus dactylopterus (Hdac) & 0.9 & 4.7 & & 2.6 \\
\hline Deania calcea (Dcal) & 0.5 & 6.7 & 2.7 & 8.6 & Holtbyrnia macrops & & 0.1 & & \\
\hline Dipturus oxyrrinchus & & & & 0.2 & Hoplostethus atlanticus & & 0.8 & & \\
\hline Etmopterus pusillus & 0.1 & & 0.1 & & Hoplostethus mediterraneus (Hmed) & 0.4 & 3.2 & 4.2 & 56.5 \\
\hline Etmopterus spinax (Espi) & 25.1 & 5.6 & 0.6 & 29.1 & Hymenocephalus italicus & 0.0 & 0.2 & & 1.0 \\
\hline Galeorhinus galeus & 0.0 & & & & Lampanyctus crocodilus (Lamp) & 0.5 & 0.6 & 1.1 & 0.3 \\
\hline Galeus atlanticus & & 0.1 & & & Lampanyctus intricarius (Lamp) & & 0.1 & 0.3 & \\
\hline Galeus melastomus (Gmel) & 10.8 & 18.3 & 9.4 & 92.4 & Lepidion eques (Lequ) & 0.1 & 12.5 & 7.9 & 27.5 \\
\hline Hexanchus griseus & 0.1 & & & & Lepidopus caudatus & 0.0 & & & \\
\hline Leucoraja circularis & 0.1 & 0.2 & 0.0 & 0.8 & Lepidorhombus boscii (Lbos) & 15.3 & 11.0 & 1.1 & 1.5 \\
\hline Leucoraja naevus & 0.0 & 0.1 & 0.0 & & Lepidorhombus whiffiagonis & 0.2 & 0.0 & 0.0 & \\
\hline Neoraja caerulea & 0.0 & 0.0 & & 0.3 & Liza aurata & 0.6 & & & \\
\hline Raja brachyura & 0.0 & & & & Lophius budegassa & 0.1 & 0.1 & & \\
\hline Raja clavata & 0.1 & 0.1 & 0.0 & & Lophius piscatorius (Lpis) & 0.8 & 0.8 & 0.5 & 0.7 \\
\hline Raja montagui & & & 0.0 & & Macroramphosus scolopax & 0.5 & & 0.1 & \\
\hline Scyliorhinus canicula & 0.4 & 0.0 & 0.1 & & Malacocephalus laevis (Mlae) & 0.6 & 0.8 & & 0.6 \\
\hline Scymnodom ringens (Srin) & 0.1 & 0.4 & 1.3 & 1.5 & Maurolicus muelleri (Mmие) & 8.9 & 5.5 & 0.2 & \\
\hline Osteichthyes & & & & & Melagnostigma atlanticum & & & 0.0 & \\
\hline Alepocephalus rostratus & & 0.0 & & 0.4 & Melanonus zugmayeri & & 0.0 & & \\
\hline Aphanopus carbo & & 0.1 & 0.0 & & Merluccius merluccius (Mmer) & 3.7 & 4.2 & 6.9 & 1.3 \\
\hline Argentina sphyraena & 0.1 & 0.2 & & & Microchirus variegatus (Mvar) & 2.7 & 0.1 & & \\
\hline Argyropelecus gigas & & & 0.0 & & Micromesistius poutassou (Мрои) & 124.9 & 48.8 & 22.7 & 5.5 \\
\hline Argyropelecus hemigymnus (Ahem) & 0.3 & 1.3 & 3.2 & & Molva macrophthalma (Mmac) & 1.2 & 0.2 & 0.2 & 1.2 \\
\hline Argyropelecus olfersii & 0.1 & 0.1 & 0.2 & 0.1 & Molva molva & & 0.1 & 0.0 & \\
\hline Arnoglossus laterna & 0.0 & & 0.0 & & Mora moro (Mmor) & 0.1 & 3.4 & 1.9 & 4.7 \\
\hline Bathysolea profundicola (Bpro) & 1.0 & 0.4 & 0.4 & 1.1 & Myctophum punctatum & & & & 0.1 \\
\hline Beryx decadactylus & 0.1 & 0.3 & & & Nemichthys scolopaceus & & & & \\
\hline Beryx splendens & & 0.1 & 0.0 & 0.1 & Neoscopelus macrolepidotus & & & & 0.1 \\
\hline Blennius ocellaris & 0.0 & & & & Nesiarchus nasutus & & 0.1 & 0.0 & 0.1 \\
\hline Callionymus lyra & 0.0 & & & & Nezumia aequalis (Naeq) & 5.1 & 35.8 & 3.2 & 37.0 \\
\hline Callionymus maculatus & 0.3 & 0.0 & 0.0 & & Notacanthus bonapartei (Nbon) & 2.0 & 2.8 & 3.0 & 0.9 \\
\hline Capros aper & 0.6 & 0.2 & 0.0 & & Notolepis rissoi & 0.0 & 0.1 & 0.0 & \\
\hline Ceratoscopelus maderensis & 0.1 & 0.2 & & & Notoscopelus kroeyerii & 0.0 & 0.1 & 0.0 & \\
\hline Chaunax pictus & & 0.1 & & & Paralepis coregonoides & & 0.0 & & \\
\hline Chelidonichthys gurnardus & & 0.2 & & & Paraliparis membranaceus & 0.0 & 0.3 & 2.5 & 0.2 \\
\hline Chlorophthalmus agassizii & 0.1 & 0.1 & & & Phycis blennoides (Pble) & 10.3 & 5.8 & 6.3 & 2.1 \\
\hline Coelorhynchus coelorhynchus (Ccoe) & 2.3 & 0.3 & & & Polyprium americanus & 0.0 & & & \\
\hline Conger conger (Ccon) & 3.0 & 1.3 & 0.5 & 0.2 & Polymetne corythaeola & & 0.7 & & 0.3 \\
\hline Cyttopsis roseus & 0.0 & & & & Raniceps raninus & & 0.0 & & \\
\hline Diaphus dumerillii & 0.0 & 0.2 & & & Scomber scombrus & 0.1 & & 0.0 & \\
\hline Echiodon dentatus & 0.9 & & & & Scorpaena scrofa & 0.0 & & & \\
\hline Electrona rissoi & 0.0 & 0.0 & & & Stomias boa & 0.1 & 0.2 & 0.1 & 0.1 \\
\hline Entelerus aequoraeus & & & 0.0 & & Synaphobranchus kaupi (Skau) & 0.1 & 4.2 & 0.3 & 1.7 \\
\hline Epigonus denticulatus & 0.0 & 0.2 & & & Trachurus mediterraneus & 0.0 & 0.1 & & \\
\hline Epigonus telescopus & & 0.2 & 0.0 & & Trachurus trachurus (Ttra) & 1.0 & 1.1 & 0.2 & 0.6 \\
\hline Facciolella oxyrhyncha & 0.0 & & & & Trachyrhynchus scabrus (Tsca) & 0.3 & 4.3 & 18.9 & 52.7 \\
\hline Gadella maraldi & 0.0 & & & & Trachyscorpia cristulata echinata (Tcri) & 0.0 & 0.1 & 0.3 & 3.6 \\
\hline Gadiculus argenteus (Garg) & 124.0 & 21.6 & 8.9 & & Trigla lyra & 0.0 & & & \\
\hline Gaidropsarus macrophthalmus (Gmac) & 2.3 & 2.0 & 0.3 & 0.4 & Xenodermichthys copei (Xcop) & 0.7 & 4.7 & 75.8 & 4.5 \\
\hline Gaidropsarus vulgaris & 0.0 & & & & Zenopsis conchifer & & 0.0 & & \\
\hline
\end{tabular}

The typifying species were selected using SIMPER analysis (which identifies the species contributing most to intra-group similarities and inter-group dissimilarities) using the same procedure as in cluster analysis, and analysing the RDA species biplot.

\section{Differences in assemblage structure}

The structure of assemblages obtained from the cluster analysis was compared. Significant differences in the mean values of the community indices (species richness, Shannon diversity, density, biomass) and trophic guilds between cluster groups were tested using a one-way ANOVA (F), under normality and homoscedasticity con- ditions, or the non-parametric alternatives (Kruskal-Wallis one-way ANOVA on ranks $[\mathrm{H}]$ ) when these conditions were not met. When significant differences were detected, pairwise a posterior tests (Student-Newman-Keuls for ANOVA and Dunn's test for Kruskal-Wallis) were run to identify the groups responsible for the differences.

\section{RESULTS}

\section{Faunal assemblages}

Both fish and invertebrates were well-represented in the samples throughout the period 1992-2006, $43.9 \%$ of the total biomass corresponding to fish and 
TABLE 2. - List of invertebrate species identified in the area. with their density (ind. ha ${ }^{-1}$ ) in each assemblage. Species codes used in Figure $3 b$ appears between brackets after species name. in those species used in RDA analysis. 0.0 mean values $>0$ and $<0.1$.

\begin{tabular}{|c|c|c|c|c|c|c|c|c|c|}
\hline & SST & USW & USE & MS & & SST & USW & USE & MS \\
\hline Crustacea & & & & & Limopsis aurita & & & & 0.1 \\
\hline Acantephyra pelagica (Apel) & & 0.3 & 0.2 & 3.5 & Loligo forbesi & 0.0 & & & \\
\hline Alpheus glaber & 0.0 & & & & Lunatia fusca & 0.0 & 0.0 & 0.0 & \\
\hline Anapagurus laevis & & 0.8 & & & Neptunea contraria & 0.8 & 0.2 & 0.0 & \\
\hline Anapagurus petiti & & 1.8 & & & Octopus salutii & 0.4 & & 0.3 & 0.1 \\
\hline Aristeus antenatus (Aant) & 0.0 & 0.6 & 0.0 & 12.0 & Opistoteuthis agassizii & 0.0 & 0.3 & 0.7 & 0.3 \\
\hline Aristomorpha foliacea & & 0.1 & 0.0 & & Pygnodontha cochlear & & 0.1 & & \\
\hline Atelecyclus rotundatus & 0.0 & 0.0 & & & Rondeletiola minor & 0.1 & & & \\
\hline Atelecyclus undecimdentatus & 0.2 & & & & Rossia macrosoma (Rmac) & 2.0 & 0.3 & 0.2 & \\
\hline Bathynectes maravigna (Bmar) & 4.1 & 1.0 & 1.9 & 0.9 & Scaphander lignarius (Slig) & 0.8 & 0.0 & & 1.6 \\
\hline Cancer bellianus & & 0.1 & & 0.1 & Semicassis saburon & 0.1 & & 0.1 & \\
\hline Chlorotocus crassicornis & 0.6 & & 0.0 & & Sepia orbignyana & 0.1 & & & \\
\hline Dichelopandalus bonnieri (Dbon) & 38.1 & 1.5 & 2.2 & 1.0 & Sepietta oweniana & 0.6 & & & \\
\hline Dorhynchus thomsoni & & 0.0 & & & Sepiola sp. & 0.7 & 0.2 & 0.0 & \\
\hline Epimeria parasitica & & 1.3 & & & Spisula subtruncata & & 0.0 & & \\
\hline Galathea intermedia & 0.0 & & 0.0 & & Todarodes sagittatus (Tsag) & 2.4 & 0.7 & 0.9 & \\
\hline Geryon trispinosus (Gtri) & 0.4 & 5.7 & 21.5 & 0.2 & Todaropsis eblanae (Tebl) & 3.6 & 3.6 & 0.5 & 0.2 \\
\hline Gnathophausia zoea (Gzoe) & & 3.0 & 1.0 & 2.1 & Troschelia berniciensis & & & 0.2 & \\
\hline Goneplax rhomboides & 0.2 & 0.1 & & & Echinodermata & & & & \\
\hline Inachus leptochirus & 0.0 & 0.1 & & & Amphiura chiajei & 0.0 & & & \\
\hline Liocarcinus depurator & 9.8 & 0.3 & 0.5 & 0.1 & Anseropoda placenta & 0.1 & 0.1 & 0.1 & 0.1 \\
\hline Liocarcinus pusillus & & 0.8 & & & Asteronyx loveni & 0.1 & & 0.1 & 0.3 \\
\hline Lophogaster typicus & & 0.0 & & & Astropecten auranticus & & & & 0.5 \\
\hline Macropipus tuberculatus (Mtub) & 21.9 & 1.0 & 0.7 & 0.2 & Astropecten irregularis (Airr) & 4.0 & 2.2 & 2.2 & 1.0 \\
\hline Macropodia longipes & 0.6 & 0.6 & 0.3 & 0.1 & Brisingella coronata & 0.3 & 0.0 & & 1.9 \\
\hline Meganyctiphanes norvegica & 0.6 & 8.9 & 6.1 & 0.5 & Brissopsis lyrifera & 34.2 & & & \\
\hline Monodaeus couchii & 0.0 & 0.2 & & & Chaetaster longipes & & 0.0 & & \\
\hline Munida intermedia (Mint) & 25.3 & 0.3 & & & Cidaris cidaris & & & 0.1 & \\
\hline Munida iris & 0.7 & 0.1 & & & Echinus acutus (Eacu) & 0.1 & 0.0 & 9.9 & 0.5 \\
\hline Munida sarsi (Msar) & 966.7 & 8.6 & 0.0 & 0.1 & Echinus esculentus & 0.0 & & & \\
\hline Munida tenuimana (Mten) & 1.5 & & 2.5 & & Echinus melo & & & 0.3 & \\
\hline Nematoscelis megalops & 0.0 & 0.0 & 2.1 & & Laetmogone violacea (Lvio) & 0.2 & & & 13.8 \\
\hline Nephrops norvegicus (Nnor) & 7.3 & 6.8 & 2.9 & 0.9 & Leptometra celtica & 0.2 & 0.7 & & \\
\hline Pagurus alatus (Pala) & 7.3 & 9.7 & 2.6 & 1.8 & Luidia ciliaris & & 0.0 & & \\
\hline Pagurus carneus & & 0.1 & & & Marthasterias glacialis & & 0.0 & & \\
\hline Pagurus excavatus (Pexc) & 1.5 & 1.1 & 0.5 & & Nymphaster arenatus & 0.3 & & 0.0 & 2.1 \\
\hline Pagurus prideaux (Ppri) & 1.9 & & & & Ophiothrix fragilis & 0.0 & 0.3 & & 0.1 \\
\hline Pandalina profunda & 0.0 & & & & Ophiura ophiura (Ooph) & 0.8 & 2.5 & 0.1 & \\
\hline Parapenaeus longirrostris & 0.0 & 0.3 & 0.0 & & Peltaster placenta & & & & 0.4 \\
\hline Pasiphaea multidentata (Pmul) & 6.9 & 28.9 & 9.4 & 0.8 & Phormosoma placenta (Ppla) & 0.2 & 16.6 & 19.0 & 29.7 \\
\hline Pasiphaea sivado (Psiv) & 118.1 & 136.1 & 94.7 & 30.3 & Porania hibrida & & & & 0.1 \\
\hline Philocheras echinulatus (Pech) & 3.1 & 4.2 & 0.8 & 0.3 & Porania pulvillus & & 0.0 & & \\
\hline Phronima sedentaria & & & 0.1 & & Pseudoporania stormii & & & & 0.1 \\
\hline Plesionika edwarsii & & 0.2 & & & Psilaster andromeda & 0.0 & & & 0.2 \\
\hline Plesionika heterocarpus & 0.4 & & 0.1 & 0.1 & Stichastrella rosea & 0.0 & & & 0.2 \\
\hline Plesionika martia (Pmar) & & 4.3 & 0.0 & 15.9 & Stichopus regalis & 0.1 & 0.2 & & \\
\hline Plesiopenaeus edwardsianus & & 0.4 & & 12.8 & Stichopus tremulus (Stre) & 3.6 & 0.6 & 0.8 & 52.4 \\
\hline Polybius henslowii & 36.1 & 188.3 & 0.5 & 58.0 & Tethyaster subinermis & 0.3 & & & \\
\hline Polycheles typhlops (Ptyp) & 4.2 & 8.9 & 4.7 & 11.0 & Thyone fusus & & & & 8.1 \\
\hline Pontophilus norvegicus (Pnor) & 0.5 & 1.4 & 4.9 & 0.5 & Brachiopoda & & & & \\
\hline Pontophilus spinosus (Pspi) & 5.9 & 1.7 & 7.2 & 2.9 & Griphus vitreus & & & & 2.2 \\
\hline Processa canaliculata (Proc) & 4.0 & 2.6 & 0.3 & 0.1 & Annelida & & & & \\
\hline Processa nouveli (Proc) & 0.6 & 0.7 & & & Chloeia venusta & 0.0 & & & \\
\hline Psathyrocaris infima & & 0.5 & & 0.4 & Laetmonice filicornis (Lfil) & 0.1 & & 0.1 & 2.8 \\
\hline Rissoides desmaresti & 0.0 & & & & Sipunculidae & & & & \\
\hline Rochinia carpenteri & & & & 0.1 & Sipunculus norvegicus & & & 0.2 & \\
\hline Scalpellum scalpellum (Ssca) & 0.2 & 0.0 & 1.7 & & Cnidaria & & & & \\
\hline Sergestes arcticus & 0.0 & 0.4 & 0.1 & & Actinauge richardi (Aric) & 4.8 & 1.3 & 0.2 & 0.1 \\
\hline Sergia robusta & & 0.0 & 0.9 & 3.9 & Adamsia carcinopados & 0.2 & & & \\
\hline Solenocera membranacea (Smem) & 10.6 & 19.5 & 0.5 & 0.2 & Aglaophenia elongata & 0.1 & 0.1 & & \\
\hline Systellaspis debilis (Sdeb) & & & 0.8 & 3.6 & Calliactis parasitica & 0.2 & & & \\
\hline Trichizostoma nicaeense & & 0.0 & & & Callogorgia verticillata & & 0.0 & & \\
\hline Mollusca & & & & & Caryophyllia smithii & 0.0 & & & \\
\hline Alloteuthis media & & 5.3 & & & Epizoanthus incrustatus (Epiz) & 10.0 & 0.7 & 0.1 & \\
\hline Argobuccinum olearium & 0.1 & 0.2 & & 0.2 & Epizoanthus paguriphilus & 0.1 & 0.1 & 0.4 & \\
\hline Bathypolipus sponsalis (Bspo) & 2.1 & 1.8 & 1.0 & 1.2 & Funiculina quadrangularis & 4.5 & & 0.2 & 0.2 \\
\hline Buccinum humphreysianum & 0.3 & 0.3 & 0.3 & & Hydractinia echinata & & 0.2 & & \\
\hline Calliostoma granulatum & & & & 0.1 & Lytocarpia myriophyllum & 0.1 & 0.1 & & 0.2 \\
\hline Charonia lampax & 0.0 & 0.1 & & & Pelagia noctiluca & 6.6 & & 9.3 & \\
\hline Colus gracilis (Colu) & 0.4 & 1.1 & 0.7 & 0.3 & Pennatula rubra & & & 0.3 & \\
\hline Corbula gibba & & 0.1 & & & Plumularia setacea & & 0.1 & & \\
\hline Cymbulia peroni & & 94.1 & & & Pteroides griseum & & 1.3 & & \\
\hline Eledone cirrhosa & 0.4 & 0.6 & 0.1 & & Siphonophora & & & & \\
\hline Galeodea rugosa & 0.3 & 0.9 & 0.3 & 0.3 & Chelophyes appendiculata & 100.9 & & 14.9 & \\
\hline Gasteropteron meckeli & 0.0 & & & & Porifera & & & & \\
\hline Hinia reticulata & 0.1 & & & & Asconema setubalense & & & & 0.1 \\
\hline Histioteuthis reversa & & 0.1 & 0.2 & 0.3 & Geodia megastrella & & & & 0.3 \\
\hline Illex coindetii & 0.3 & 0.3 & 0.7 & & Phakelia ventilabrum & 0.0 & 0.1 & & \\
\hline
\end{tabular}




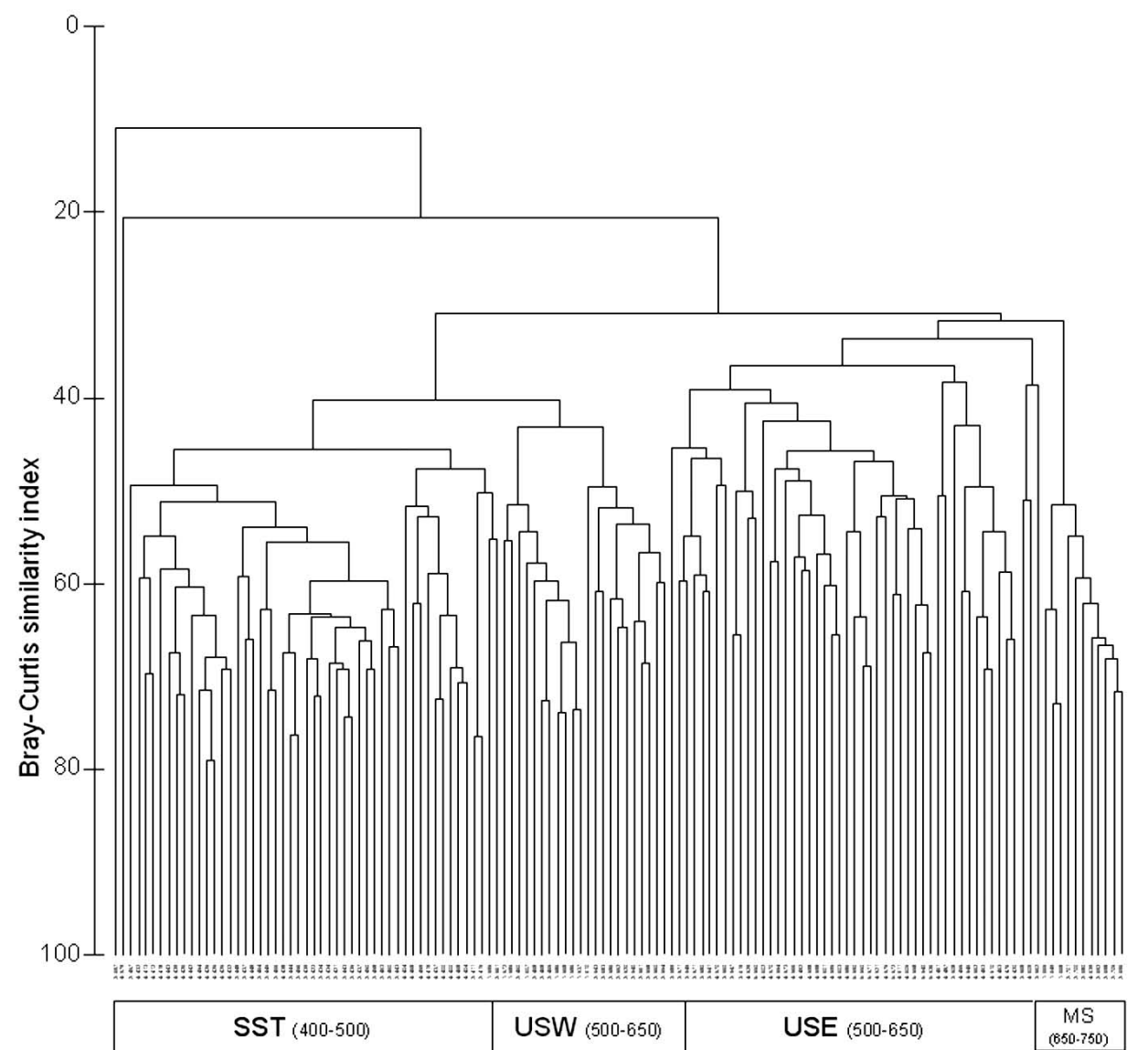

FIG. 2. - Dendrogram showing the clustering of hauls from the fish and invertebrate species matrix (1992-2006).

$56.1 \%$ to invertebrates. A total of 251 species were identified: 104 fish (19 chondrichthyes and 85 osteichthyes, Table 1) and 147 invertebrates (61 crustaceans, 33 molluscs, 30 echinoderms, 15 cnidarians, and 8 species corresponding to 5 other phyla, Table 2). The most abundant fish species in the whole area were blue whiting (Micromesistius poutassou) (25.5\%), silvery pout (Gadiculus argenteus) (22.9\%), Xenodermichthys copei (6.3\%), blackmouth catshark (Galeus melastomus) (5.7\%), Etmopterus spinax (5.1\%) and Chimaera monstrosa $(4.7 \%)$. The dominant invertebrate species were Munida sarsi (58.4\% of total numbers), the shrimps Pasiphaea sivado (14.9\%), Dichelopandalus bonnieri (2.4\%) and Pasiphaea multidentata (1.7\%), and the urchin Brissopsis lyrifera (2.1\%). The cluster analysis indicated that assemblages were conditioned mainly by depth and geographical position, with the 500 and 650 $\mathrm{m}$ isobaths and the vertical of the Cape Peñas boundaries determining faunal distribution patterns.

Those factors together accounted for the division into four main faunal assemblages (Fig. 2): a shelfslope transition assemblage (SST) grouping the shallowest hauls (400-500 m depth) without geographical differentiation; two upper slope assemblages (500-650 $\mathrm{m})$, one corresponding to the western of Cape Peñas area (USW) and one to the eastern area (USE); and a middle slope group clustering the deepest hauls (MS,
650-750 m, all hauls located west of Cape Peñas, except one). The first dichotomy separated a group clustering the SST and USW assemblages from a group clustering the USE and MS groups. Therefore, the upper slope west of Cape Peñas is more similar to the shallower areas than to the upper slope east of the cape, this latter assemblage being more similar to the middle slope assemblage. Hence, Cape Peñas is a barrier to species distribution, though only for upper slope species, not for shallower ones (MS hauls were mostly west of Cape Peñas).

\section{Environmental variables explaining species distribution}

RDA results for species densities are listed in Table 4. The full model, which included all the variables and the factor "year" as covariable, was highly significant $(P=0.001)$. The RDA applied separately for each environmental factor as variable and the others as covariable showed that all of them were significant except Temperature, Salinity and Year. Depth was the main factor affecting the species composition, accounting for $18.1 \%$ of the explained variance (EV). Below Depth, the factor Longitude is the second key variable $(9.5 \%$ $\mathrm{EV}$ ) followed by sedimentary characteristics: the presence of medium and fine sands (MFS) and silts, and the 
TABLE 3. - Results of the SIMPER analysis applied to the cluster groups on log-transformed fish and invertebrate. The table shows the contribution (Contrib.\%) and cumulative (Cum.\%) percentage of the species that most contribute to the intragroup Bray-Curtis similarity and intergroup dissimilarities, together with the $\mathrm{N}$ of each group.

\begin{tabular}{|c|c|c|c|c|c|c|c|}
\hline Intragroup similarity & & & & Intergroup dissimilarity & & & \\
\hline SST d: 46.9 & $\mathrm{~N}$ & Contrib\% & Cum\% & SST/USW d: 63.6 & N SST & N USW & Cum\% \\
\hline M. poutassou & 124.9 & 13.4 & 13.4 & G. argenteus & 124.0 & 21.6 & 4.7 \\
\hline G. argenteus & 124.0 & 11.8 & 25.2 & M. sarsi & 966.7 & 8.6 & 9.2 \\
\hline M. sarsi & 966.7 & 6.7 & 31.9 & N. aequalis & 5.1 & 35.8 & 13.2 \\
\hline D. bonnieri & 38.1 & 6.6 & 38.5 & P. sivado & 118.1 & 136.1 & 17.0 \\
\hline L. boscii & 15.3 & 6.5 & 45.0 & D. bonnieri & 38.1 & 1.5 & 20.4 \\
\hline USW d: 41.5 & & & & SST/USE d: 70.7 & N SST & N USE & Cum\% \\
\hline P. sivado & 136.1 & 9.9 & 9.9 & G. argenteus & 124.0 & 8.9 & 5.2 \\
\hline M. poutassou & 48.8 & 8.4 & 18.3 & M. sarsi & 966.7 & 0.0 & 9.8 \\
\hline N. aequalis & 35.8 & 8.3 & 26.6 & X. copei & 0.7 & 75.8 & 13.4 \\
\hline S. kaupi & 4.2 & 7.5 & 34.1 & G. trispinosus & 0.4 & 21.5 & 16.8 \\
\hline H. dactylopterus & 4.7 & 5.1 & 39.2 & D. bonnieri & 38.1 & 2.2 & 20.1 \\
\hline USE d: 43.0 & $\mathrm{~N}$ & Contrib\% & Cum\% & USW/USE d:69.8 & N USW & N USE & Cum\% \\
\hline P. sivado & 94.7 & 14.6 & 14.6 & N. aequalis & 35.8 & 3.2 & 4.3 \\
\hline M. poutassou & 22.7 & 8.7 & 23.3 & P. sivado & 136.1 & 94.7 & 8.1 \\
\hline G. trispinosus & 21.5 & 8.1 & 31.4 & C. monstrosa & 16.2 & 2.0 & 11.7 \\
\hline X. copei & 75.8 & 7.2 & 38.6 & G. trispinosus & 5.7 & 21.5 & 15.3 \\
\hline E. acutus & 9.9 & 7.1 & 45.7 & M. poutassou & 48.8 & 22.7 & 18.3 \\
\hline MS d: 54.3 & $\mathrm{~N}$ & Contrib\% & Cum\% & MS/USW d: 66.7 & N MS & N USW & Cum\% \\
\hline L. eques & 27.5 & 8.8 & 8.8 & T. scabrus & 52.7 & 4.3 & 4.0 \\
\hline C. monstrosa & 53.5 & 8.3 & 17.1 & S. tremulus & 52.4 & 0.6 & 7.4 \\
\hline T. scabrus & 52.7 & 7.6 & 24.7 & G. melastomus & 92.4 & 18.3 & 10.8 \\
\hline G. melastomиs & 92.4 & 7.6 & 32.3 & H. mediterraneus & 56.5 & 3.2 & 14.2 \\
\hline \multirow[t]{7}{*}{ N. aequalis } & 37.0 & 6.9 & 39.2 & $P$. sivado & 30.3 & 136.1 & 17.5 \\
\hline & & & & MS/USE d: 64.2 & N MS & N USE & Cum $\%$ \\
\hline & & & & C. monstrosa & 53.5 & 2.0 & 4.6 \\
\hline & & & & N. aequalis & 37.0 & 3.2 & 8.6 \\
\hline & & & & T.scabrus & 52.7 & 18.9 & 12.5 \\
\hline & & & & H. mediterraneus & 56.5 & 4.2 & 16.2 \\
\hline & & & & S. tremulus & 52.4 & 0.8 & 19.9 \\
\hline
\end{tabular}

organic matter content $(\% \mathrm{OM})$. These analyses did not detect significant variations in the species composition between the different surveys.

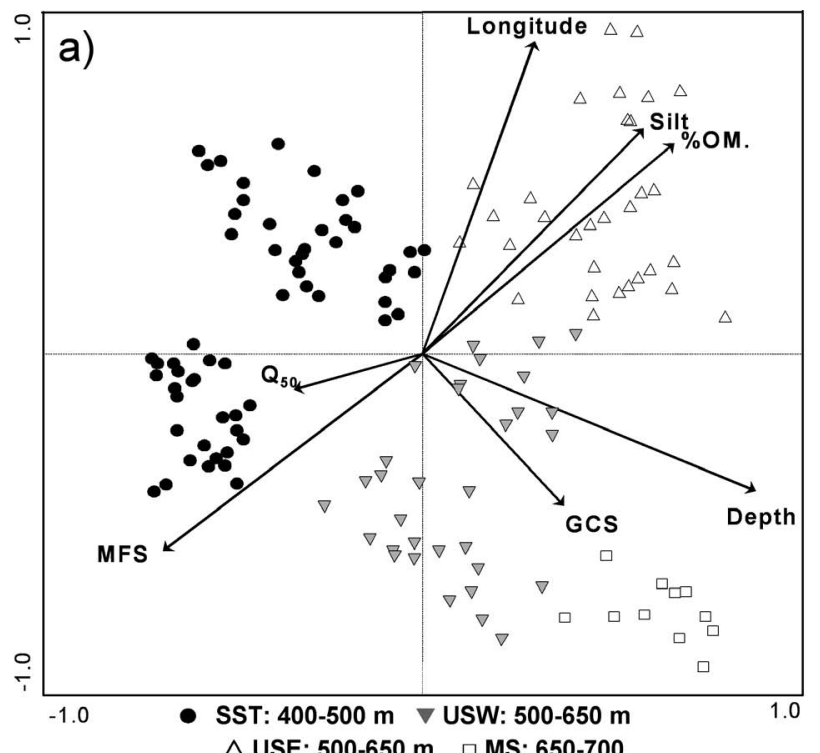

Using the RDA model on each cluster group as variable and all the environmental factors as covariables, all groups were significant (Table 4). RDA biplot (Fig.

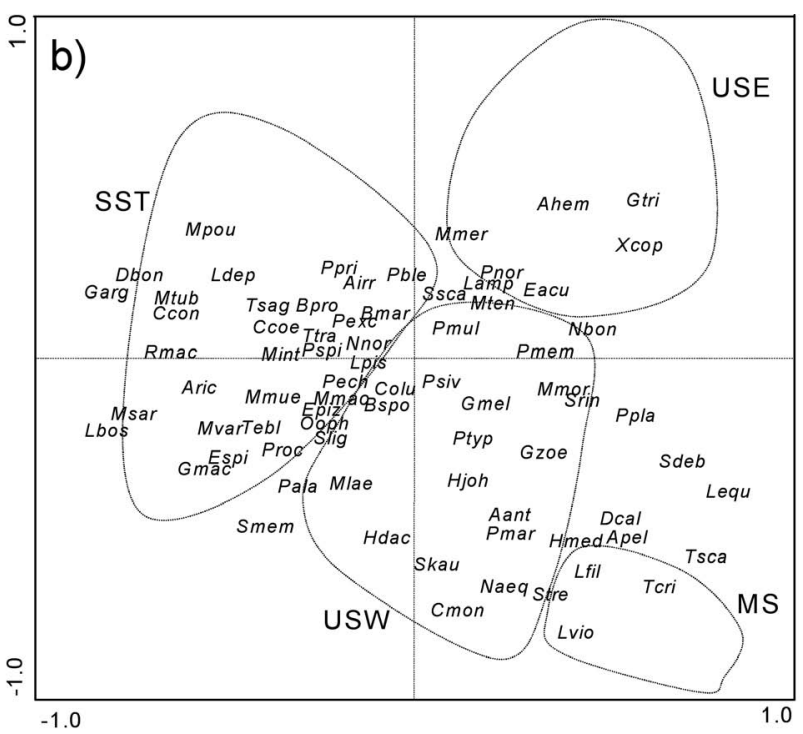

FIG. 3. - RDA biplots. a) Environmental variables versus hauls. Symbols represent cluster groups of hauls: SST, shelf-slope transition; USW, western upper slope; USE, eastern upper slope; MS, middle slope. $\mathrm{Q}_{50}$, median particle diameter; $\mathrm{S}_{0}$, sorting coefficient; GCS, weight percentage of gravel and coarse sands $(>500 \mu \mathrm{m})$; MFS = weight percentage of medium, fine and very fine sands $(63-500 \mu \mathrm{m})$; Silt, percentage weight of silt $(<63 \mu \mathrm{m}) ; \% \mathrm{OM}$, weight percentage of organic matter. b) Species. Areas represent groups of hauls. Species codes figure in Tables 2 and 3. 
TABLE 4. - Results of the RDA for the species density matrix. The explained variance $(\mathrm{EV})$, the F-statistic and its significance $(P$-value) for both the full model, which contains all the variables included in the model, and each individual variable after extracting the effect of the covariables is also indicated. Note that the sum of the EV for all variables is greater than $100 \%$ due to the shared variance.

\begin{tabular}{|c|c|c|c|c|}
\hline Effect & Covariable & EV & $F$-ratio & $P$-value \\
\hline \multicolumn{5}{|c|}{ Variables included in the RDA } \\
\hline Full model & Year $(\mathrm{Y})$ & $41.3 \%$ & 9.68 & 0.001 \\
\hline Depth (D) & $\mathrm{Y}, \mathrm{T}, \mathrm{S}, \mathrm{Q}, \mathrm{G}_{1,2,3}, \mathrm{O}, \mathrm{L}$ & $18.1 \%$ & 8.82 & 0.001 \\
\hline Temperature $(\mathrm{T})$ & $\mathrm{Y}, \mathrm{D}, \mathrm{S}, \mathrm{Q}, \mathrm{G}_{1,2,3}, \mathrm{O}, \mathrm{L}$ & $0.6 \%$ & 1.21 & 0.202 \\
\hline Salinity (S) & $\mathrm{Y}, \mathrm{D}, \mathrm{T}, \mathrm{Q}, \mathrm{G}_{1,2,3}, \mathrm{O}, \mathrm{L}$ & $0.4 \%$ & 0.73 & 0.810 \\
\hline $\mathrm{Q}_{50}(\mathrm{Q})$ & $\mathrm{Y}, \mathrm{D}, \mathrm{T}, \mathrm{S}, \mathrm{G}_{1,2,3}, \mathrm{O}, \mathrm{L}$ & $5.7 \%$ & 4.39 & 0.001 \\
\hline $\operatorname{GCS}\left(\mathrm{G}_{1}\right)$ & $\mathrm{Y}, \mathrm{D}, \mathrm{T}, \mathrm{S}, \mathrm{Q}, \mathrm{G}_{2,3}, \mathrm{O}, \mathrm{L}$ & $5.6 \%$ & 5.17 & 0.001 \\
\hline $\operatorname{MFS}\left(\mathrm{G}_{2}\right)$ & $\mathrm{Y}, \mathrm{D}, \mathrm{T}, \mathrm{S}, \mathrm{Q}, \mathrm{G}_{1,3}, \mathrm{O}, \mathrm{L}$ & $7.9 \%$ & 7.07 & 0.001 \\
\hline Silt $\left(\mathrm{G}_{3}\right)$ & $\mathrm{Y}, \mathrm{D}, \mathrm{T}, \mathrm{S}, \mathrm{Q}, \mathrm{G}_{1,2}, \mathrm{O}, \mathrm{L}$ & $7.8 \%$ & 6.98 & 0.001 \\
\hline$\% \mathrm{OM}(\mathrm{O})$ & $\mathrm{Y}, \mathrm{D}, \mathrm{T}, \mathrm{S}, \mathrm{Q}, \mathrm{G}_{1,2,3}, \mathrm{~L}$ & $7.9 \%$ & 7.61 & 0.001 \\
\hline Longitude (L) & $\mathrm{Y}, \mathrm{D}, \mathrm{T}, \mathrm{S}, \mathrm{Q}, \mathrm{G}_{1,2,3}, \mathrm{O}$ & $9.5 \%$ & 3.53 & 0.002 \\
\hline Year $(\mathrm{Y})$ & $\mathrm{D}, \mathrm{T}, \mathrm{S}, \mathrm{Q}, \mathrm{G}_{1,2,3}, \mathrm{O}, \mathrm{L}$ & $0.7 \%$ & 1.39 & 0.091 \\
\hline \multicolumn{5}{|c|}{ Variables not included in the RDA } \\
\hline Cluster S & $\mathrm{Y}, \mathrm{D}, \mathrm{T}, \mathrm{S}, \mathrm{Q}, \mathrm{G}_{1,2,3}, \mathrm{O}, \mathrm{L}$ & $5.1 \%$ & 6.29 & 0.001 \\
\hline Cluster USW & $\mathrm{Y}, \mathrm{D}, \mathrm{T}, \mathrm{S}, \mathrm{Q}, \mathrm{G}_{1,2,3}, \mathrm{O}, \mathrm{L}$ & $3.6 \%$ & 2.59 & 0.003 \\
\hline Cluster USE & $\mathrm{Y}, \mathrm{D}, \mathrm{T}, \mathrm{S}, \mathrm{Q}, \mathrm{G}_{1,2,3}, \mathrm{O}, \mathrm{L}$ & $3.7 \%$ & 2.42 & 0.003 \\
\hline Cluster MS & $\mathrm{Y}, \mathrm{D}, \mathrm{T}, \mathrm{S}, \mathrm{Q}, \mathrm{G}_{1,2,3}, \mathrm{O}, \mathrm{L}$ & $4.5 \%$ & 2.69 & 0.003 \\
\hline
\end{tabular}

3a) shows the relationships between depth and sediment characteristics. The SST group is associated with sediments consisting mainly of sand (medium and fine sand), but with finer particles in the eastern part, covered by sand in the western part and covered by silt in the eastern part. This geographical/sedimentary pattern was clearer on the upper slope, where the two cluster groups were evident. The USW hauls located west of Cape Peñas were characterized by medium and fine sands, while in the USE hauls silt predominated and there was a higher organic content. Finally, deeper hauls grouped in the cluster group MS (650-700 m) were characterized by gravel and coarse sands (Fig. 3a).

\section{Characterizing species}

Faunal differences between assemblages and autoecological information of the species inhabiting the different environments were obtained by combining the results from the Simper and the RDA analyses (Table 3, Fig. 3b), and the density data (Tables 1,2). Blue whiting, the most abundant species in the study area, is one of the typifying species in all assemblages except the MS (Table 3), showing a decrease in density with depth and greater abundance in the SST and west of Cape Peñas than to the east (Table 1). For this reason, this species is located in the negative segment of RDA axis 1, related to the SST group and on the positive side of axis 2, related to the western area (Fig. 3b). Ubiquitous species such as the blackmouth catshark Galeus melastomus and the glass shrimp Pasiphaea sivado are located near the RDA centroid (Fig. 3).

The shallower assemblage (SST) was typified by the silvery pout (G. argenteus), the shrimp Dichelopandalus bonnieri, the squat lobster (Munida sarsi) and the four-spotted megrim (Lepidorhombus boscii) (Table 3, Fig. 3b). The abundance of M. sarsi in the SST was almost 1000 ind./ha, compared to the $c a .10$ ind./ha found on the USW (Tables 2, 3). In addition to these typifying species, inside the SST group there was a group of species related to shallower depths, most of them having a wide distribution on the shelf, such as the eel Conger conger, the crabs Macropipus tuberculatus and Liocarcinus depurator, the stout bobtail (Rossia macrosoma) the starfish Astropecten irregularis, or the sea pen Funiculina quadrangularis (Tables 1,2).

Upper slope assemblages were both typified by ubiquitous species such as $P$. sivado and G. melastomus (Table 3). Below these species, the USW was typified mainly by fish: Kaup's arrowtooth eel (Synapobranchus kaupi), the blackbelly rosefish (Helicolenus dactylopterus) and the grenadier Nezumia aequalis (Table 3, Fig. 3b). Table 3 and Fig. 3b show that the hermit crab (Pagurus alatus) and the shrimp Solenocera membranacea were invertebrate species related to the USW. Hauls located east of Cape Peñas (USE) were typified by the crab Geryon trispinosus, the bluntsnout smooth-head (Xenodermichthys copei), the hatchet fish (Argyropelecus hemygimnus) and the sea urchin Echinus acutus.

Finally, the assemblage grouping middle slope hauls (MS) was typified by Chimaera monstrosa, the codling Lepidion eques, the grenadiers Trachyrhynchus scabrus and N. aequalis, the Atlantic thornyhead (Trachyscorpia cristulata), the Mediterranean slimehead (Hoplostethus mediterraneus), the sea cucumbers Stichopus tremulus and Laetmogone violacea, the worm Laetmonice filicornis, the red shrimps Aristeus antennatus and Plesionika martia, and the oplophorid shrimps Systellaspis debilis and Acanthephyra pelagica (Tables 1, 2; Fig. 3b), together with the ubiquitous G. melastomus.

TABLE 5. - Significance tests between cluster groups for a) community indices (S: species richness; H: Shannon diversity, N: density; $\mathrm{W}$ : biomass, of fish and invertebrates), b) trophic guilds of fish, and c) trophic guilds of invertebrates. 1, SST; 2, USW; 3, USE; 4, MS.

\begin{tabular}{|c|c|c|c|}
\hline \multicolumn{4}{|l|}{ a) Community indices } \\
\hline S fish & $\mathrm{F}=15.7$ & $P<0.0001$ & 4213 \\
\hline $\mathrm{S}$ inv. & $\mathrm{H}=12.2$ & $P=0.0470$ & $\overline{214} \overline{3}$ \\
\hline H fish & $\mathrm{H}=26.8$ & $P<0.0001$ & 2431 \\
\hline $\mathrm{H}$ inv. & $\mathrm{F}=2.3$ & $P=0.0776$ & $\bar{*}$ \\
\hline $\mathrm{N}$ fish & $\mathrm{H}=35.7$ & $P<0.0001$ & $142 \underline{3}$ \\
\hline $\mathrm{N}$ inv. & $\mathrm{H}=15.8$ & $P=0.0012$ & $\underline{1 \overline{4} 2} \overline{3}$ \\
\hline W fish & $\mathrm{H}=16.5$ & $P=0.0009$ & 4213 \\
\hline W inv. & $\mathrm{H}=7.4$ & $P=0.0312$ & $1 \overline{243}$ \\
\hline \multicolumn{4}{|l|}{ b) Fish trophic guilds } \\
\hline Benthos-feeders & $\mathrm{H}=23.1$ & $P<0.0001$ & $\underline{4} \underline{231}$ \\
\hline Fish-feeders & $\mathrm{H}=5.4$ & $P=0.1428$ & $-\frac{*}{*}$ \\
\hline Plankton-feeders & $\mathrm{H}=37.0$ & $P<0.0001$ & $\underline{1234}$ \\
\hline \multicolumn{4}{|c|}{ c) Invertebrate trophic guilds } \\
\hline Benthos-feeders & $\mathrm{H}=13.7$ & $P=0.0033$ & $132 \underline{4}$ \\
\hline Deposit-feeders & $\mathrm{H}=29.7$ & $P<0.0001$ & $4 \underline{12} \underline{3}$ \\
\hline Suspension-feeders & $\mathrm{H}=30.4$ & $P<0.0001$ & $2 \underline{43}$ \\
\hline Zooplankton-feeders & $\mathrm{H}=16.6$ & $P=0.0009$ & $\underline{4} 213$ \\
\hline
\end{tabular}


a)

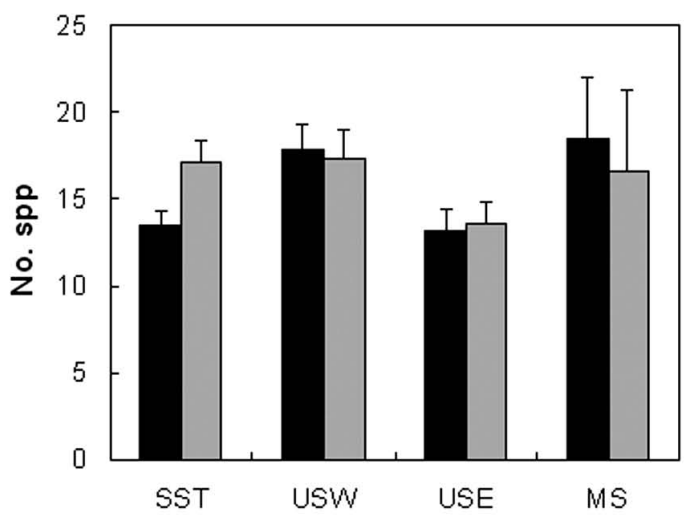

c)

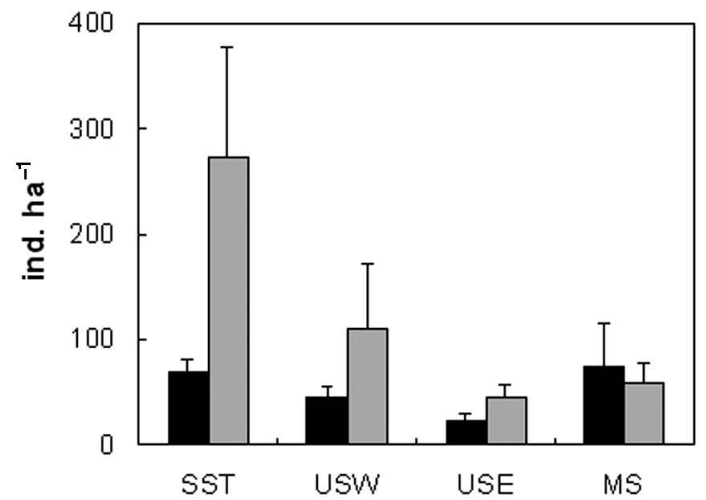

b)

Shannon diversity

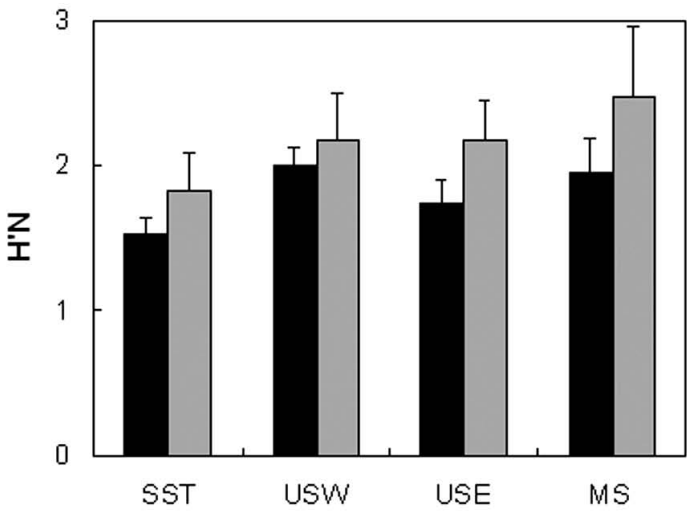

d)

Biom ass

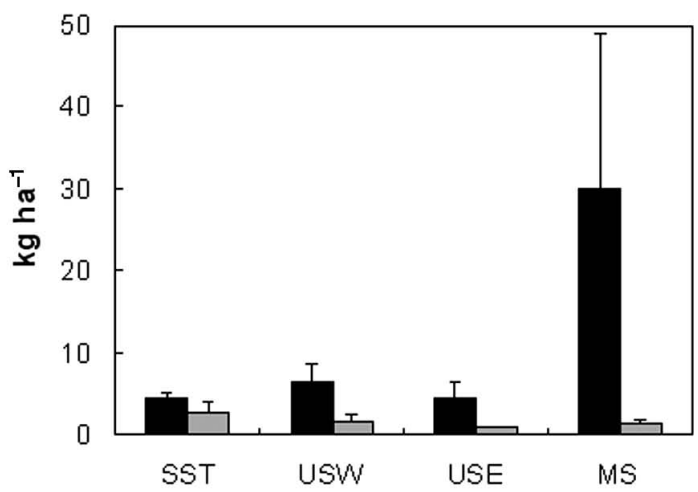

FIG. 4. - Differences in community indices between cluster groups for fish (black) and invertebrates (grey).

a)

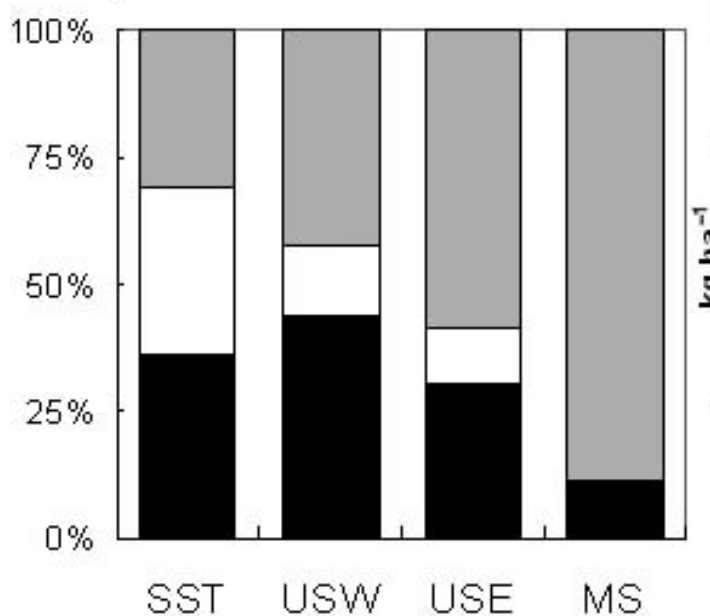

Fish trophic guilds

b)

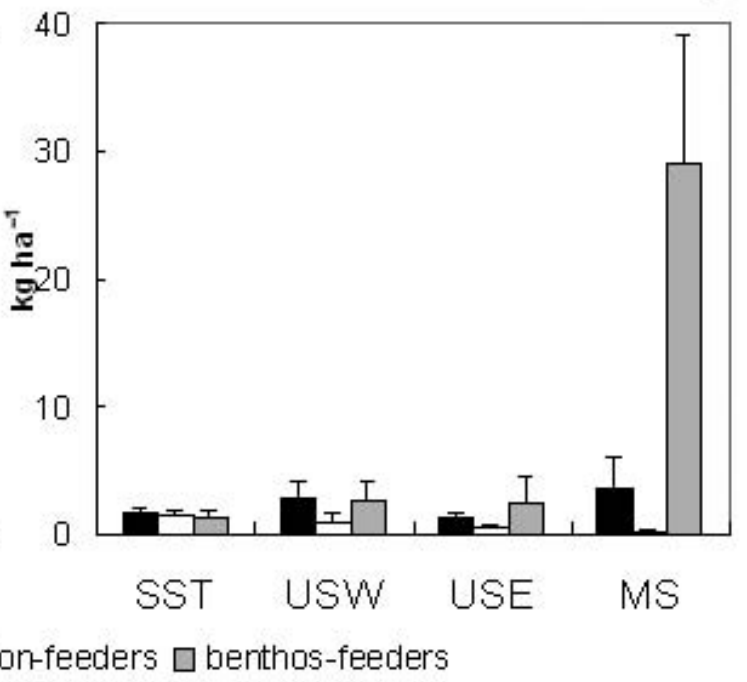

FIG. 5. - Fish species trophic guild composition in the assemblages in terms of biomass percentage (a) and mean biomass (b).

\section{Differences in assemblage structure}

The significance test (Table 5) shows differences between assemblages for all indices, except for H' of invertebrates. The MS and the USW showed higher fish species richness than the SST and the USE (Fig. 4a). For both fish and invertebrates, the USE was significantly poorer than the other assemblages. In Shannon diversity, no clear patterns were found, the only significant difference being the greater diver- 
a)

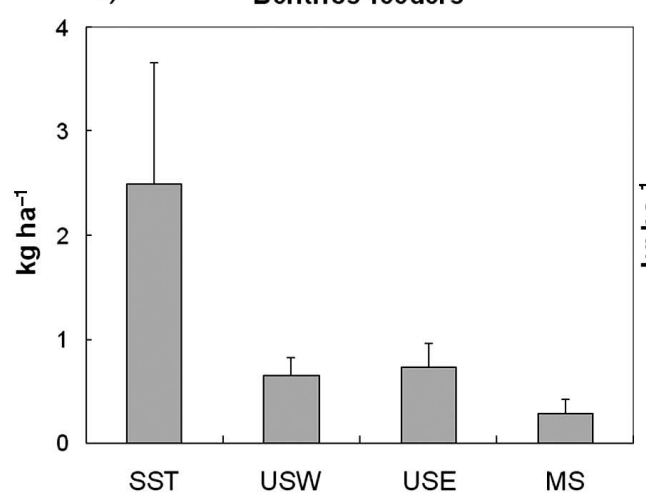

C) Suspension-feeders

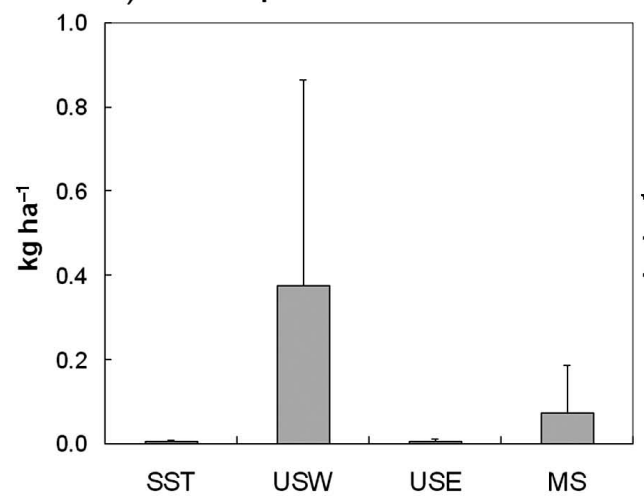

b)

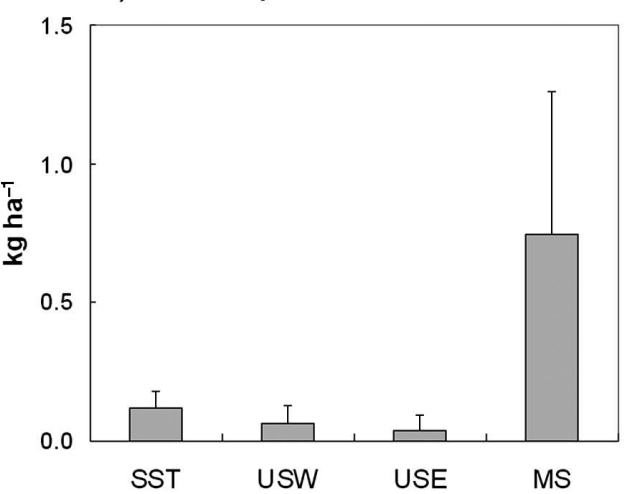

d) Zooplankton-feeders

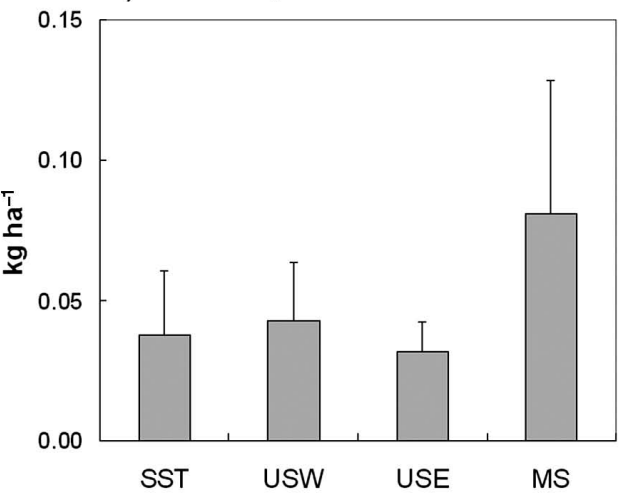

FIG. 6. - Invertebrate species trophic guild composition in the cluster groups in terms of biomass.

sity of fish on the USW than in the SST (Fig. 4b, Table 5).

The lowest fish densities were found on the USE (Fig. 4c, Table 5). Considering invertebrate species, the assemblage with the highest density was clearly the SST, with more than 250 ind./ha (mainly due to the high densities of the squat lobster $M$. sarsi). The most outstanding result regarding biomass was the highest fish biomass found on the MS (Fig. 4d). Invertebrate biomass was higher in the SST (again for the squat lobster).

\section{Differences in trophic structure among assemblages}

There was a clear difference in trophic structure among the assemblages (Fig. 5). The percentage of piscivorous fishes was lower in the SST and on the MS than in the upper slope groups (Fig 5a), although these differences were not significant in terms of biomass (Table 5b). The percentage of plankton-feeding fish decreased with depth: they were found in high abundance in the SST, medium abundance on the upper slope, and relatively low abundance on the MS. Biomass of plankton-feeding fish in the SST was significantly higher than that in the other deeper assemblages. Benthic-feeding fish showed the opposite pattern, with the lowest values in the shallower SST and the highest

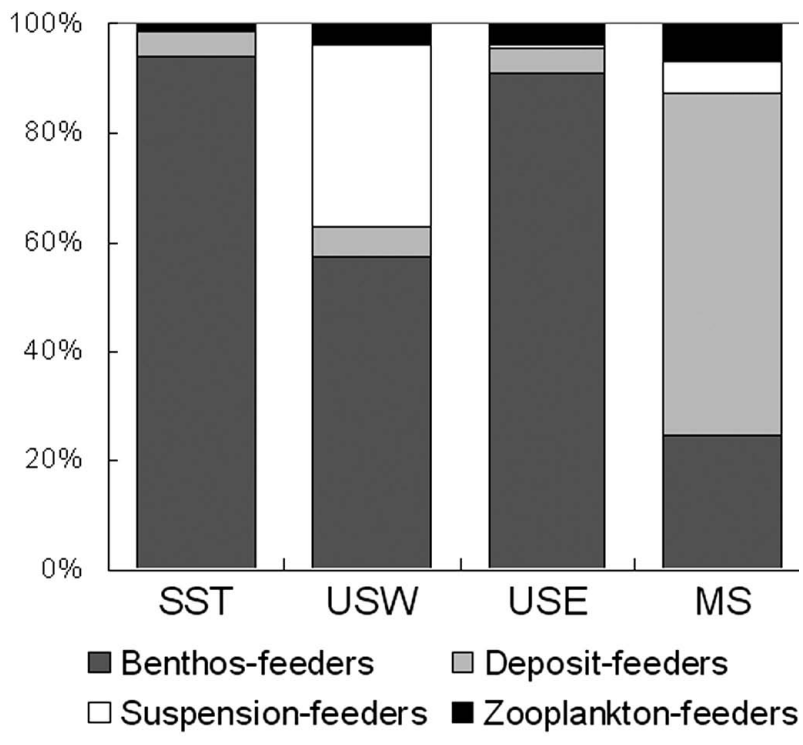

FIG. 7. - Invertebrate species trophic guild composition in the cluster groups in percentage.

values on the MS. This trophic guild showed significantly higher biomass on the MS (Fig. 5b, Table 5b).

Invertebrates also showed differences in trophic structure among assemblages (Figs. 6 and 7). Benthosfeeding invertebrates were more abundant in the SST, 
the lowest values being found on the MS. Deposit feeders were clearly more abundant on the MS, where higher densities of holothurids such as Stichopus tremulus and Laetmogone violacea were found (Table 2). A higher dominance of filter-feeders was found on the USW, and of zooplankton-feeders on the MS (mainly oplophorid shrimps). In terms of percentages (Fig. 7), benthos-feeders were clearly dominant in the SST and on the USE. This trophic guild was also dominant on the USW, although a higher percentage of filter-feeders was also present. In contrast, the MS was clearly dominated by deposit- and zooplankton-feeders.

\section{DISCUSSION}

The present study describes the existence of faunal discontinuities in relation to geographical and depth boundaries. A geographic faunal boundary was found for slope communities at Cape Peñas, between the Bay of Biscay and the Atlantic Ocean, but only for the upper slope, not for the shelf-slope transition area. For instance, the western Cape Peñas upper slope assemblage shows more similarities with the shallower shelf-slope transition assemblage than the assemblage dwelling at the same depths in the eastern part. This is a very interesting result since it is not very common to find "barriers" in deeper, more stable, communities (e.g. Cartes et al, 2002; Carney, 2005), and these boundaries have been mainly related to depth rather than geographical gradients (Cartes et al., 2009).

In the present study, we also found faunal boundaries at two isobaths, 500 and $650 \mathrm{~m}$, showing a discontinuity in the distribution of invertebrates and fish. Carney (2005) reported slope fauna to be distributed in depth bands, mainly in response to range restriction due to pressure and food availability. In a comparative study among several areas, Carney identified the $500 \mathrm{~m}$ depth as a zone of high species turnover that was common in all the upper slopes analysed (Carney, 2005). The depths of 500 and $650 \mathrm{~m}$ were also cited as faunal boundaries for decapod crustaceans by Cartes $e t$ al. (2007) on the Le Danois Bank (central Cantabrian Sea), and for all invertebrates in the western Mediterranean Sea (Cartes et al., 2009).

Environmental differences between the western and eastern areas of Cape Peñas consist mainly in a higher frequency and intensity of upwelling events to the west of the cape, reflected in all production processes (Lavín et al., 2005; Gil, 2008). In addition, topography differs greatly between these two areas, since east of Cape Peñas the Cantabrian sea shelf is very narrow and has an abrupt shelf break, whereas west of Cape Peñas the shelf is wider $(25-75 \mathrm{~km})$ and has a gradual transition between shelf and slope (Rey and Medialdea, 1989). Our results confirm that these environmental differences lead to significant faunal differences. The narrowness of the eastern shelf, with an abrupt shelf break and a steeper slope, where the depth and depth-related gradients ( $T, S$, nutrients, etc.) are sharper, produces a higher overlap in the bathymetric distribution of species east of Cape Peñas. This may be the explanation for the greater similarities between the USE and MS assemblages in comparison with the SST and USW assemblages, which has less sharp gradients.

Nevertheless, longitudinal gradients in production seem to be more important than topographic differences. The enhancement of primary production processes above the shelf (Gil, 2008) causes the predominance of planktophagous fishes such as blue whiting in the shallowest assemblage (shelf-slope transition). This preponderance is more evident west of Cape Peñas, where upwelling is stronger and the pelagic environment is richer in nutrients (Gil, 2008). A similar trend was described by Cartes et al. (2002), with an increase in planktophagous fishes in the Alboran Sea in comparison with northern areas of the Mediterranean, related to geographical differences in primary production. All these facts are reflected in inter-assemblage differences in ecological indices: western areas are richer in species as a consequence of more complex food webs and in western assemblages the abundance of trophic guilds related to water column production is higher (plankton-feeding fishes and filter- and zooplanktonfeeding invertebrates). The shelf-slope transition assemblage has a higher biomass but is less diverse than the deeper assemblages, as a consequence of the dominance of plankton-feeders (Sánchez and Serrano, 2003). Cartes et al. (1994) described the same pattern of peaks of biomass and low diversity in the shelf-slope transition zone in the Mediterranean, whereas in the same area Abelló et al. (1988) found a higher diversity of decapod crustaceans on the middle slope. The lowest diversity in the shallowest assemblage and the highest in the deepest one are also in agreement with the increase in diversity with depth described in several papers (Hessler and Sander, 1967; Sanders, 1968; Stuart et al., 2003).

We observed an increase in invertebrate depositfeeders below $650 \mathrm{~m}$ on the middle slope, the predominant species being the holothurids Stichopus tremulus and Laetmogone violacea, and the omnivorous urchin Phormosoma placenta. This dominance of depositfeeding echinoderms in deeper areas of the slope has already been described (e.g. Sibuet, 1977; Thurston et al., 1994; Iken et al., 2001; Cartes et al., 2009). Zooplankton-feeders, and among them mainly Aristeus antennatus and oplophorid shrimps, are also predominant under $700 \mathrm{~m}$ (Cartes et al., 2002, 2007). Cartes et al. (2002) suggest a higher dependence on pelagic trophic resources by invertebrate bathyal communities. These trophic guild patterns are generally consistent with the published data on distribution of zooplankton and macrobenthos as available preys (Sabatés et al., 1989; Cartes et al., 2002, 2007; Sánchez et al., 2008).

An opposite pattern was found between fish and invertebrate trophic guilds in our study, since plankton-feeding fish were dominant in the SST whereas zooplankton-feeding invertebrates were dominant on 
the middle slope, nevertheless benthos feeding fish dominate in MS and benthos-feeding invertebrates in SST. Cartes et al. (1994) reported mesopelagic crustaceans to be predominant on the slope whereas benthopelagic fishes were predominant on the shelf. Prey availability on the middle slope is probably insufficient to sustain the huge biomass of planktonfeeding fishes such as blue whiting (Preciado et al., 2008), and fish feeding mainly on benthos (Preciado et al., 2009).

Most of the typifying species in the area have been already cited as key species in deep sea studies in other areas (Gordon, 1986; Haedrich and Merrett, 1988; Cartes et al., 2002; Mytilineou et al., 2005; Madurell et al., 2004). Blue whiting is one of the most abundant fish in midwater ecosystems in the northeastern Atlantic, living mostly at depths between 100 and $600 \mathrm{~m}$, although its range may extend from surface waters to beyond 1000 m depth (Heino and Godø, 2002; Hátún et al., 2007). This eurybathic species is dominant in the habitats studied and has been described as one of the keystone species for the Cantabrian Sea food web (Libralato et al., 2005; Preciado et al., 2008, 2009). Moranta et al. (2007) stated that the maximum abundances of $N$. aequalis and T. scabrus occur below $600 \mathrm{~m}$ in the Mediterranean Sea. This fact could explain the affinity of these two grenadiers for deeper assemblages.

The typifying invertebrate species were the glass shrimps Pasiphaea sivado, Munida sarsi and Dichelopandalus bonnieri in the shallower SST. This is in agreement with previous works (Abelló et al., 1988; Cartes et al., 1994). Munida sarsi is one of the characteristic species of the SST (Fariña et al., 1997), being replaced by Munida tenuimana on deeper grounds (Cartes et al., 2007). The bathymetric affinities of other crustaceans such as D. bonnieri, Geryon trispinosus and the oplophorid shrimps Acantephyra pelagica and Systellaspis debilis agree with the description of Le Danois Bank by Cartes et al. (2007), and the same occurs with the echinoderms mentioned in the present work (Sánchez et al., 2008; Cartes et al., 2009).

The general conclusion that can be drawn is that the presumed stability of deep-sea fauna is only apparent (Sanders, 1968; Stuart et al., 2003; Cartes et al., 1994), and that pronounced geographical changes in megafaunal assemblages interact with bathymetrical bands. Geographical differences in production processes, together with depth, seem to be the key factors explaining fish and invertebrate fauna distribution on the northern Iberian slope.

\section{ACKNOWLEDGEMENTS}

This study was possible thanks to the invaluable work of all the participants in the North of Spain bottom trawl surveys and the crew of the RV Cornide de Saavedra since 1983 . We are very much indebted to Dr. Izaskun Preciado for her valuable comments on an earlier version of the manuscript.

\section{REFERENCES}

Abelló, P., F.J. Valladares and A. Castellón. - 1988. Analysis of the structure of decapod crustacean assemblages off the Catalan coast (North-West Mediterranean). Mar. Biol., 98: 39-49.

Buchanan, J.B. - 1984. Sediment analysis. In: N.A. Holme, A.D. McIntyre (eds.), Methods for the Study of Marine Benthos, pp. 41-65. Blackwell Scientific Publications, Oxford.

Carney, R.S. - 2005. Zonation of deep biota in continental margins. Oceanogr. Mar. Biol. Annu. Rev., 43: 211-278.

Cartes, J.E., P. Abelló, D. Lloris, A. Carbonell, P. Torres, F. Maynou and L. Gil de Sola. - 2002. Feeding guilds of western Mediterranean demersal fish and crustaceans: analysis based on a spring survey. Sci. Mar., 66(2): 209-220.

Cartes, J.E., J.B. Company and F. Maynou. - 1994. Deep-water decapod crustacean communities in the Northwestern Mediterranean: influence of submarine canyons and season. Mar. Biol., 157(1): 221-229.

Cartes, J.E., F. Maynou, E. Fanelli, C. Romano, V. Mamouridis and V. Papiol. - 2009. The distribution of megabenthic, invertebrate epifauna in the Balearic Basin (western Mediterranean) between 400 and $2300 \mathrm{~m}$ : Environmental gradients influencing assemblages composition and biomass trends. J. Sea Res., 61: 244-257.

Cartes, J.E., A. Serrano, F. Velasco, S. Parra and F. Sánchez. - 2007. Community structure and dynamics of deep-water decapod assemblages from Le Danois Bank (Cantabrian Sea, NE Atlantic): Influence of environmental variables and food availability. Prog. Oceanogr., 75: 797-816.

Dayton, P.K. and R.R. Hessler. - 1972. Role of biological disturbance in maintaining diversity in the deep sea. Deep-Sea Res., 19: 199-208.

Fariña, C., J. Freire. and E. González-Gurriarán. - 1997. Megabenthic decapod crustacean assemblages on the Galician continental shelf and upper slope (north-west Spain). Mar. Biol., 127: 419-434.

Gage, J.D. - 2003. Food inputs, utilization, carbon flow and energetics. In: Tyler, P.A. (ed.), Ecosystems of the Deep Ocean. Ecosystems of the World 28, pp 313-380. Elsevier, Amsterdam.

Gordon, J.D.M. - 1986. The Fish Populations of the Rockall Trough. Proc. Royal. Soc. Edin. - Series B, 88: 191-207.

Gil, J. - 2008. Macro and mesoscale physical patterns in the Bay of Biscay. J. Mar. Biol. Ass. U.K., 88(2): 217-225.

Grassle, J.F. and N.J. Maciolek. - 1992. Deep-sea species richness: regional and local diversity estimates from quantitative bottom samples. Am. Nat., 139(2): 313-341.

Haedrich R. L. and N.R. Merrett. - 1988. Summary atlas of deepliving demersal fishes in the North Atlantic Basin. J. Nat. Hist., 22: 1325-1362.

Hátún, H., J. Arge and A. Sandø. - 2007 Environmental influence on the spawning distribution and migration pattern of northern blue whiting (Micromesistius poutassou). ICES CM 2007/B:06.

Heino, M. and O.R. Godø. - 2002. Blue whiting - a key species in the mid-water ecosystems of the north-eastern Atlantic. ICES CM 2002/L:28.

Hessler, R.R. and H.L. Sanders. - 1967. Faunal diversity in the deep sea. Deep-Sea Res., 14: 65-78.

Iken, K., T. Brey, U. Wand, J. Voigt and P. Junghans. - 2001. Food web structure of the benthic community at the Porcupine Abyssal Plain (NE Atlantic): a stable isotope analysis. Prog. Oceanogr., 50(1-4): 383-405.

Jongman, R.H.G., C.J.F. Ter Braak and O.F.R. Van Tongeren. 1987. Data analysis in community and landscape ecology. Pudoc Wageningen, 286.

Lavín, A., L. Valdés, F. Sánchez, P. Abaunza, A. Forest, J. Boucher, P. Lazure and A.M. Jegou. - 2005. The Bay of Biscay: the encountering of the ocean and the shelf. In: A.R. Robinson and K.H. Brink (eds.), The Sea, vol. 14, part B, ch. 24: 935-1002. Harvard University Press, Cambridge.

Libralato, S., V. Christensen and D. Pauly. - 2005. A method for identifying keystone species in food web models. Ecol. Model., 195: 153-171.

López-Jamar, E., R.M. Cal, G. González, R.B. Hanson, J. Rey, G. Santiago and K.R. Tenore. - 1992. Upwelling and outwelling effects on the benthic regime of the continental shelf off Galicia, NW Spain. J. Mar. Res., 50: 465-488. 
Madurell, T., J.E. Cartes and M. Labropoulou. - 2004. Changes in the structure of fish assemblages in a bathyal site of the Ionian Sea (eastern Mediterranean). Fish. Res., 66: 245-260.

Moranta, J., E. Massutí, M. Palmer and J.D.M. Gordon. - 2007. Geographic and bathymetric trends in abundance, biomass and body size of four grenadier fishes along the Iberian coast in the western Mediterranean. Prog. Oceanogr., 72(1): 63-83.

Mytilineou, C., C-Y. Politou, C. Papaconstantinou, S. Kavadas, G. D'Onghia and L. Sion. - 2005. Deep-water fish fauna in the Eastern Ionian Sea. Belg. J. Zool., 135: 229-233.

Piñeiro, C.G., M. Casas R. and Bañón. - 2001. The deep-water fisheries exploited by Spanish fleets in the Northeast Atlantic: a review of the current status. Fish. Res., 51: 311-320.

Preciado, I., F. Velasco, and I. Olaso. - 2008. The role of pelagic fish as forage for the demersal fish community in the southern Bay of Biscay. J. Mar. Syst., 72: 407-417.

Preciado, I., J.E. Cartes, A. Serrano, F. Velasco, I., Olaso, F. Sánchez and I. Frutos. - 2009 Resource utilisation by deep-sea sharks at the Le Danois Bank, Cantabrian Sea, northeast Atlantic Ocean. J. Fish Biol., 75: 1331-1355.

Rex, M.A., C.T. Stuart, R.R. Hessler, J.A. Allen, H.L. Sanders and G.D.F. Wilson. - 1993. Global-scale latitudinal patterns of species diversity in the deep-sea benthos. Nature, 365: 636-639.

Rey, J. and T. Medialdea. - 1989. Los sedimentos cuaternarios superficiales del margen continental español. Publ. Esp. Ins. Esp. Oceanogr., 3.

Sabatés, A. and J.M. Gili and F. Pagès, 1989. Relationship between zooplankton distribution, geographic characteristics and hydrographic patterns off the Catalan coast (Western Mediterranean). Mar. Biol.: 103, 153-159.

Sánchez, F. and A. Serrano. - 2003. Variability of groundfish communities of the Cantabrian Sea during the 1990s. ICES Mar. Sci. Symp., 219: 249-260.

Sánchez, F., A. Serrano, S. Parra, M. Ballesteros and J.E. Cartes. 2008. Habitat characteristics as determinant of the structure and spatial distribution of epibenthic and demersal communities of Le Danois Bank (Cantabrian Sea, N. Spain). J. Mar. Syst., 72: 64-86.

Sanders, H.L. - 1968. Marine benthic diversity: a comparative study. Am. Nat., 102 (925): 243-282.

Serrano, A., F. Sánchez and G. García-Castrillo. - 2006. Epibenthic communities of trawlable grounds of the Cantabrian Sea. Sci. Mar., 70S1: 149-159.

Serrano, A., I. Preciado, E. Abad, F. Sánchez, S. Parra and I. Frutos. - 2008. Spatial distribution patterns of demersal and epibenthic communities on the Galician continental shelf (NW Spain). $J$. Mar. Syst., 72: 87-100.

Sibuet, M. - 1977. Repartition et diversité des Echinoderms (Holothurides- Astérides) en zone profonde dans le Golfe de Gascogne. Deep-Sea Res., 24: 549-563.

Sorbe, J.C. - 1999. Deep-sea macrofaunal assemblages within the benthic boundary layer of the Cap-Ferret Canyon (Bay of Biscay, NE Atlantic). Deep-Sea Res. II, 46: 2309-2329.

Stuart, C.T., M.A. Rex and R.J. Etter. - 2003. Large-scale spatial and temporal patterns of deep-sea benthic species diversity. In: P.A. Tyler (ed.), Ecosystems of the Deep Ocean. Ecosystems of the World 28, pp. 293-311. Elsevier, Amsterdam.

Thurston, M., Bett, B.; Rice, A., Jackson, P., 1994. Variations in the invertebrate abyssal megafauna in the North Atlantic Ocean. Deep-Sea Res. I, 41, 9: 1321-1348.

Verdonschot, P.F.M. and C.J.F. Ter Braak. - 1994. An experimental manipulation of oligochaete communities in mesocosms treated with chlorophytos or nutrient additions: multivariate analyses with Monte Carlo permutation tests. Hydrobiologica, 278: 251-266.

Scient. ed.: M.P. Olivar.

Received January 28, 2010. Accepted November 15, 2010.

Published online April 8, 2011. 